\title{
Vitamin K2 stimulates MC3T3-E1 osteoblast differentiation and mineralization through autophagy induction
}

\author{
WEIWEI LI ${ }^{1}$, SHAOKUN ZHANG ${ }^{1}$, JIE LIU ${ }^{2}$, YONGYI LIU ${ }^{1}$ and QINGWEI LIANG $^{3}$ \\ ${ }^{1}$ Department of Orthopedics, The First Hospital of China Medical University, Shenyang, Liaoning 110000; \\ ${ }^{2}$ Science Experiment Center of China Medical University, Shenyang, Liaoning 110122; \\ ${ }^{3}$ Department of Sports Medicine, The First Hospital of China Medical University, Shenyang, Liaoning 110000, P.R. China
}

Received April 29, 2018; Accepted March 6, 2019

DOI: $10.3892 / \mathrm{mmr} .2019 .10040$

\begin{abstract}
Vitamin K2 likely exerts its protective effects during osteoporosis by promoting osteoblast differentiation and mineralization. However, the precise mechanism remains to be fully elucidated. Autophagy maintains cell homeostasis by breaking down and eliminating damaged proteins and organelles. Increasing evidence in recent years has implicated autophagy in the development of osteoporosis. The aim of the present study was to verify whether vitamin K2 (VK2) can induce autophagy during the differentiation and mineralization of osteoblasts. In the present study, MC3T3-E1 osteoblasts were treated with various doses of VK2 $\left(10^{-8}-10^{-3}\right.$ M) for 1-5 days. The results revealed no cytotoxicity at concentrations below $10^{-5} \mathrm{M}$, but cell viability was reduced in a dose-dependent manner at concentrations above $10^{-5} \mathrm{M}$. Furthermore, MC3T3-E1 osteoblasts were seeded in 6-well plates in complete medium supplemented with dexamethasone, $\beta$-glycerophosphate and vitamin C (VC) for osteogenic differentiation. MC3T3-E1 osteoblasts treated with different concentrations $\left(10^{-5}, 10^{-6}\right.$ and $\left.10^{-7} \mathrm{M}\right)$ of VK2 for $24 \mathrm{~h}$ on days 1 , 3,5 and 7 of the differentiation protocol. It was confirmed that VK2 promoted osteoblast differentiation and mineralization by using alkaline phosphatase (ALP) and alizarin red staining. Using western blotting, immunofluorescence, monodansylcadaverine staining and reverse transcription-quantitative polymerase chain reaction, it was observed that VK2 induced autophagy in osteoblasts. The results revealed that VK2 $(1 \mu \mathrm{M})$ significantly increased ALP activity and the conversion of microtubule associated protein 1 light chain 3- $\alpha$ (LC3) II to LC3I in MC3T3-E1 osteoblasts $(\mathrm{P}<0.05)$ at every time point. The number of fluorescent bodies and the intensity increased with $\mathrm{VK} 2$, and decreased following treatment with $3-\mathrm{MA}+\mathrm{VK} 2$. There was an increase in the mRNA expression
\end{abstract}

Correspondence to: Professor Qingwei Liang, Department of Sports Medicine, The First Hospital of China Medical University, 155 North Nanjing Street, Shenyang, Liaoning 110000, P.R. China E-mail: cmulqw@163.com

Key words: osteoporosis, osteogenesis, vitamin K2, autophagy levels of ALP, osteocalcin (OCN) and Runt-related transcription factor 2 in VK2-treated cells $(\mathrm{P}<0.01)$. The present study further confirmed the association between autophagy and osteoblast differentiation and mineralization through treatment with an autophagy inhibitor [3-methyladenine (3-MA)]. Osteoblasts treated with 3-MA exhibited significant inhibition of ALP activity and osteogenic differentiation (both $\mathrm{P}<0.05$ ). In addition, ALP activity and osteogenesis in the VK2+3-MA group was lower compared with VK2-treated cells $(\mathrm{P}<0.05$ for both). The present study confirmed that VK2 stimulated autophagy in MC3T3 cells to promote differentiation and mineralization, which may be a potential therapeutic target for osteoporosis.

\section{Introduction}

Osteoporotic fractures are increasingly gaining attention due to their high incidence rates and economic burden (1). Osteoporosis is a pathological condition characterized by a decrease in bone mineral density or bone mass, which can reduce bone strength and lead to fractures. Clinical osteoporosis is classified into two types: Type I, or postmenopausal osteoporosis; and type II, i.e., osteoporosis in the elderly $(1,2)$. Bone homeostasis depends on the synergistic activities of osteoclasts and osteoblasts, and any imbalance between bone formation and resorption may lead to a number of diseases, including osteoporosis, Paget's bone disease and osteodystrophy (3). Apart from decreased bone mass, osteoporosis is associated with a slower rate of bone differentiation following an increase in the number of adipocytes and osteoclasts, decreased osteoblasts and enhanced bone resorption $(4,5)$. In clinical terms, the prevention of osteoporosis is particularly significant and includes resistance exercises to increase bone density, adequate calcium in the diet, and calcium supplement medications (6). In recent years, vitamin K2 (VK2) has attracted attention as an auxiliary drug for preventing osteoporosis (7).

VK2 is a fat-soluble vitamin that is structurally similar to other members of the same family, including phylloquinone, menaquinones and menadione (7). It is a critical factor in the blood clotting process (8-10), and epidemiological studies have demonstrated that a lack of VK2 may lead to osteoporosis and osteoarthritis in older individuals $(10,11)$. In addition, VK2 
has been clinically utilized to prevent osteoporosis, and likely exerts its protective effects by promoting osteoblast differentiation and mineralization (12-14). However, the precise mechanism remains to be fully elucidated (7).

Increasing evidence in recent years has implicated autophagy in the development of osteoporosis. Autophagy is an essential process that maintains homeostasis in eukaryotic cells by breaking down and eliminating damaged proteins and organelles $(15,16)$. When a cell is subjected to external stress, it may initiate autophagy to adjust to the associated stimulus by degrading cytoplasmic substances to provide energy for cell survival $(17,18)$. Autophagy has a critical role in physiological conditions, and is associated with certain diseases, including cancer, diabetes and leukemia. A lack of autophagy in osteoblasts can decrease mineralizing capacity, and induce an imbalance in the population of osteoblasts and osteoclasts, resulting in a low bone mass phenotype (19). The aim of the present study was to verify whether VK2 is able to promote the differentiation and mineralization of osteoblasts by inducing autophagy.

\section{Materials and methods}

Chemical reagents. $\alpha$-Modification minimum essential medium ( $\alpha$-MEM) was purchased from HyClone (GE Healthcare Life Sciences, Logan, UT, USA). Fetal bovine serum (FBS) was acquired from Clark Bioscience (Claymont, DE, USA). The MTT Cell Proliferation and Cytotoxicity Assay kit was purchased from Nanjing Keygen Biotech Co., Ltd. (Nanjing, China). The Alkaline Phosphatase (ALP) Assay kit was purchased from Nanjing Jiancheng Bioengineering Institute (Nanjing, China). VK2, vitamin C (VC), and dexamethasone (Dex) were acquired from Sigma-Aldrich (Merck KGaA, Darmstadt, Germany). $\beta$-Glycerophosphate and alizarin red were purchased from Beijing Solarbio Science \& Technology Co., Ltd. (Beijing, China). The autophagy inhibitor 3-methyladenine (3-MA) and agonist rapamycin were purchased from Selleck Chemicals (Houston, TX, USA). Rabbit anti- microtubule associated protein 1 light chain 3- $\alpha$ (LC3)b antibody was purchased from Abcam (Cambridge, UK; cat. no. ab192890). Rabbit anti- $\beta$-actin antibody and goat anti-rabbit IgG antibody were purchased from BIOSS (Beijing, China; cat. nos. bs-0061R and bs-0295G, respectively). Anti-beclin 1 antibody was purchased from Cell Signaling Technology, Inc. (Danvers, MA, USA; cat. no. 3738S) and Cy3-conjugated Affinipure Goat Anti-Rabbit IgG $(\mathrm{H}+\mathrm{L})$ was obtained from Wuhan Sanying Biotechnology (Wuhan, China; cat. no. SA00009-2).

Cell culture and differentiation. MC3T3-E1 is a preosteoblast cell line. It may be used to study differential and mineral induction in an experimental system that mimics a bone-like extracellular matrix (ECM) environment. The appearance of the ECM is crucial to the bone formation process. The mouse cranial osteoblast (MC3T3-E1 Subclone 14; American Type Culture Collection CRL-2594) cell line was obtained from Cell Bank of the Type Culture Collection of the Chinese Academy of Sciences (Shanghai, China). A subclone with high differentiation potential was derived from the parental cell line for studying critical events during osteoblast differentiation and mineralization. The cells were seeded in $15 \mathrm{~cm}^{2}$ flasks in $\alpha$-MEM containing $1 \%$ antibiotics and 10\% FBS (complete medium), and the medium was changed every 2 days. Cells were passaged when they reached $90 \%$ confluence. For osteogenic differentiation, the osteoblasts were first seeded in 6-well plates at a density of $1 \times 10^{4}$ cells/well in complete medium and cultured for 3 days until the cells reached $70 \%$ confluence. To initiate the differentiation, $3 \mathrm{ml}$ complete medium supplemented with Dex $\left(10^{-7} \mathrm{M}\right), \beta$-glycerophosphate $(10 \mathrm{mM})$ and $\mathrm{VC}(50 \mu \mathrm{g} / \mathrm{ml})$ was added to each well, and the differentiation medium was replaced every 2 days.

Cell viability assay. The cell viability assay was performed to analyze the possible cytotoxic effects of different concentrations of VK2 $\left(10^{-8}-10^{-3} \mathrm{M}\right)$ on MC3T3-E1 cells. The MC3T3-E1 osteoblasts were seeded into 96-well plates. When $70 \%$ confluence was achieved, cells were treated with VK2 at different concentrations $\left(10^{-8}-10^{-3} \mathrm{M}\right)$ for different durations (1-5 days). At least six replicated wells per sample were prepared. The cell viability was measured at $48 \mathrm{~h}$ using the MTT Cell Proliferation and Cytotoxicity Assay kit, according to the manufacturer's protocol.

Quantitative assay of ALP activity. MC3T3-E1 osteoblasts were plated in 6-well plates at a density of $2.5 \times 10^{5}$ cells/well and treated with different concentrations $\left(10^{-5}, 10^{-6}\right.$ and $\left.10^{-7} \mathrm{M}\right)$ of the drugs (3MA, VK2 and 3MA+VK2; untreated controls were also included) for $24 \mathrm{~h}$ during the differentiation protocol, and harvested after 1, 3, 5 and 7 days. Total protein was extracted from the cells using radioimmunoprecipitation assay (RIPA) buffer [300 mM NaCl, $50 \mathrm{mM}$ Tri-HCL (pH 7.6), $0.5 \%$ Triton X-100, $2 \mathrm{mM}$ phenylmethylsulfonyl fluoride] and quantified using the bicinchoninic acid (BCA) assay. ALP activity was measured using a fluorescence detection kit per the manufacturer's protocol. The standard curve was plotted using p-nitrophenol, and the ALP activity of each sample was normalized to the total protein concentration.

Mineralization analysis. MC3T3-E1 osteoblasts were treated with the different drugs as described above, during the 7-day differentiation. On the 7th day, the supernatant was discarded, and the cells were washed twice with PBS and fixed with $4 \%$ paraformaldehyde at room temperature $\left(20^{\circ} \mathrm{C}\right)$ for $5 \mathrm{~min}$. Calcium nodes were stained with $0.1 \%$ alizarin red at $37^{\circ} \mathrm{C}$ for $1 \mathrm{~h}$. For quantitative analysis, cells were destained with ethylpyridinium chloride for $30 \mathrm{~min}$ at room temperature and transferred to a 96-well plate to measure the absorbance at $550 \mathrm{~nm}$ using a microplate reader.

Western blotting. MC3T3-E1 osteoblasts were seeded into $100 \mathrm{~mm}$ dishes and cultured in complete $\alpha$-MEM to $70 \%$ confluency, following which the cells were treated with the different drugs as described above. VK2 was used at the optimal concentration of $10^{-6} \mathrm{M}(1 \mu \mathrm{M})$. Total proteins were extracted following lysis of the cells in RIPA buffer at $4^{\circ} \mathrm{C}$ for $1 \mathrm{~h}$. The lysates were centrifuged at 20,000 x g for $30 \mathrm{~min}$, and the protein concentration was determined using a BCA protein quantification kit. Equal amounts of protein $(30 \mu \mathrm{g})$ were loaded into each well of a 7.5-15\% SDS-PAGE gel and 


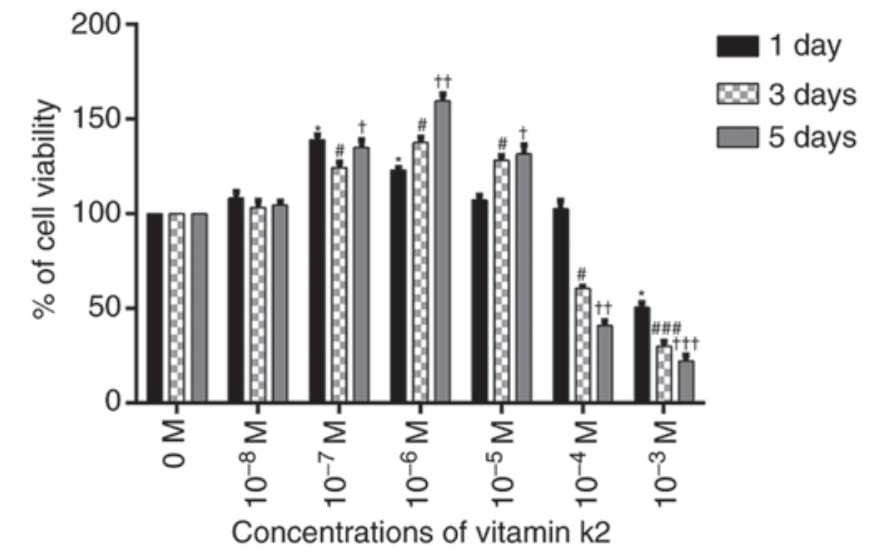

Figure 1. Cytotoxic effect of VK2 on MC3T3-E1 cells. Different concentrations of VK2 $\left(10^{-8}-10^{-3} \mathrm{M}\right)$ were used to stimulate the MC3T3-E1 cells for 1-5 days. Concentrations of VK2 between $10^{-7}-10^{-5} \mathrm{M}$ did not cause cytotoxicity. However, concentrations above $10^{-5} \mathrm{M}$ significantly reduced cell viability in a dose-dependent manner. Compared with the control, the concentration of $10^{-8} \mathrm{M}$ had no significant effect on the cell. The experimental data are expressed as the mean \pm standard deviation. Significance analysis of the experimental data for each group was performed using one-way analysis of variance and Tukey's multiple comparisons test. " $\mathrm{P}<0.05$ vs. control group at 1 day; ${ }^{\#} \mathrm{P}<0.05,{ }^{\# \# \#} \mathrm{P}<0.001$ vs. control group at 3 days; ${ }^{\dagger} \mathrm{P}<0.05,{ }^{\dagger \dagger} \mathrm{P}<0.01$, ${ }^{\dagger \dagger} \mathrm{P}<0.001$ vs. control group at 5 days. VK2, vitamin K2.

separated by electrophoresis. The separated protein bands were transferred to a polypropylene fluoride membrane and, following incubation overnight at $4^{\circ} \mathrm{C}$ with primary antibody against LC3b $(1: 2,000)$, beclin $1(1: 1,000)$ and $\beta$-actin $(1: 2,000)$, respectively. Next, the membrane was incubated with goat anti-rabbit secondary antibody $(1: 5,000)$ for $2 \mathrm{~h}$ at room temperature. The specific bands were visualized using an enhanced chemiluminescence detection system (MF-ChemiBIS 3.2, DNR Bio-Imaging Systems, Ltd., Neve Yamin, Israel) and imaged with an Alpha Imager HP (ProteinSimple, San Jose, CA, USA). The band density was quantified using the ImageJ image processing program (National Institutes of Health, Bethesda, MD, USA. software version $1.5 b)$.

Immunofluorescence assay. MC3T3-E1 osteoblasts were seeded in 24-well plates and upon reaching 50\% confluency, were treated with 3-MA, VK2 and VK2+3MA for $1 \mathrm{~h}$. The cells were washed twice with PBS, fixed with $4 \%$ paraformaldehyde at room temperature for $15 \mathrm{~min}$, and permeabilized with $0.5 \%$ Triton X-100 for 2 min at room temperature. After blocking for $2 \mathrm{~h}$ with $5 \%$ bovine serum albumin (cat. no. ST023-50 g, Beyotime Institute of Biotechnology, Shanghai, China) at room temperature, the cells were incubated overnight with anti-LC3b antibody $(1: 200)$ at $4^{\circ} \mathrm{C}$. After $1 \mathrm{~h}$ incubation with Cy3-conjugated secondary antibody $(1: 3,000)$, the cells were counterstained with DAPI for $10 \mathrm{~min}$ at room temperature. The stained cells were imaged using confocal fluorescence microscopy (magnification, x200, DS-U3 Nikon Eclipse CI; Nikon Corporation, Tokyo, Japan).

Monodansylcadaverine (MDC) staining. The cells were seeded in 24-well plates at a density of $4.0 \times 10^{4}$ cells/well, and VK2 $(1 \mu \mathrm{M})$ and the autophagy inhibitor 3-MA $(0.5 \mathrm{mM})$ were added. The supernatant was removed and after washing the cells once, they were stained with MDC (a fluorescent marker of autophagic vacuoles) for $45 \mathrm{~min}$ at $37^{\circ} \mathrm{C}$. The stained cells were imaged using an Olympus fluorescence microscope (magnification, x200, IX71; Olympus Corporation, Tokyo, Japan).

Reverse transcription-quantitative polymerase chain reaction $(R T-q P C R)$. MC3T3-E1 osteoblasts were seeded at a density of $5 \times 10^{4}$ cells/well in a 6 -well plate for 3 days until the cells reached $70 \%$ confluence. The cells were treated with the different drugs as described, and total RNA was extracted using TRIzol ${ }^{\circledR}$ reagent (Invitrogen; Thermo Fisher Scientific, Inc., Waltham, MA, USA), according to the manufacturer's protocol. A total of $\sim 1 \mu \mathrm{g}$ of RNA was used in the reverse transcription reaction using RevertAid First Strand cDNA Synthesis Kit (Thermo Fisher Scientific, Inc.) according to the manufacturer's protocols. For gene-specific primed cDNA synthesis, the reaction was conducted for $60 \mathrm{~min}$ at $42^{\circ} \mathrm{C}$. Then, for random hexamer primed synthesis, incubate for $5 \mathrm{~min}$ at $25^{\circ} \mathrm{C}$. RT was terminated by heating at $70^{\circ} \mathrm{C}$ for $5 \mathrm{~min}$. Real-time PCR was performed using the SYBR Green PCR Master Mix (Applied Biosystems; Thermo Fisher Scientific, Inc.) on the ABI 7500 Fast Real-Time PCR System (Applied Biosystems 7500 System Sequence Detection System, software version 2.6.2; Thermo Fisher Scientific, Inc.). The PCR conditions were as follows: Initial denaturation at $95^{\circ} \mathrm{C}$ for $15 \mathrm{~min}$, followed by 40 cycles of denaturation at $94^{\circ} \mathrm{C}$ for $20 \mathrm{sec}$, annealing at $60^{\circ} \mathrm{C}$ for $30 \mathrm{sec}$ and extension at $72^{\circ} \mathrm{C}$ for $60 \mathrm{sec}$. The primer sequences were as follows: Runt related transcription factor 2 (Runx2) forward, 5'-CCCTGAACT CTGCACCAAGT-3'; Runx2 reverse, 5'-TGGAGTGGA TGGATGGGGAT-3'; osteocalcin (OCN) forward, 5'-AGC AGCTTGGCCCAGACCTA-3'; OCN reverse, 5'-TAGCGC CGGAGTCTGTTCACTAC-3'; $\beta$-actin forward, 5'-TTC GTTGCCGGTCCACACCC-3'; and $\beta$-actin reverse, 5'-GCT TTGCACATGCCGGAGCC-3'.

Statistical analysis. All experiments were repeated at least three times and the results are expressed as the mean \pm standard deviation. Statistical analysis was performed using SPSS 13.0 software (SPSS, Inc., Chicago, IL, USA). Different groups were compared by one-way analysis of variance followed by Tukey's multiple comparisons test. $\mathrm{P}<0.05$ was considered to indicate a statistically significant difference.

\section{Results}

VK2 is not cytotoxic to MC3T3-E1 cells at concentrations below $10^{-5} \mathrm{M}$. An MTT assay was used to analyze the possible cytotoxic effects of different concentrations of VK2 $\left(10^{-8}-10^{-3} \mathrm{M}\right)$ on MC3T3-E1 cells. The cells were stimulated for different durations (1-5 days). No cytotoxicity was observed at concentrations below $10^{-5} \mathrm{M}$, but cell viability was reduced in a dose-dependent manner at concentrations above $10^{-5} \mathrm{M}$. Furthermore, compared with the control group, $10^{-8} \mathrm{M}$ VK2 did not have a significant effect on the induction of differentiation and mineralization. Therefore, all subsequent experiments were performed with VK2 at concentrations between $10^{-7}$ and $10^{-5} \mathrm{M}$ (Fig. 1). 

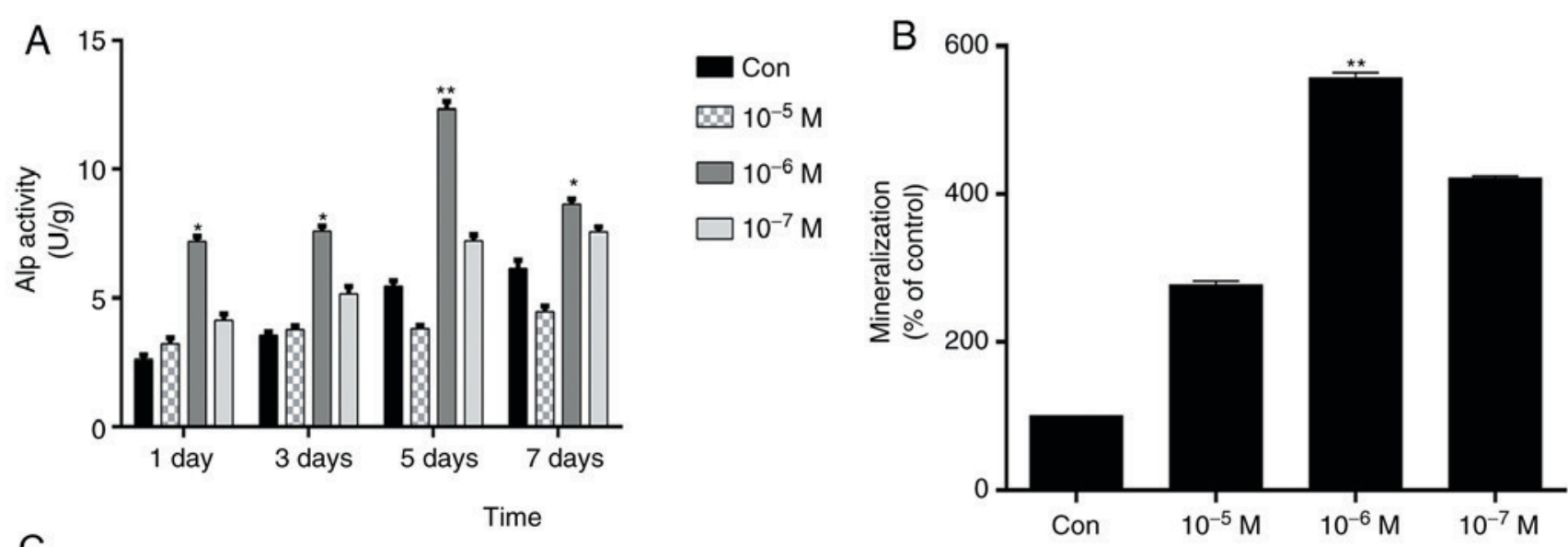

C

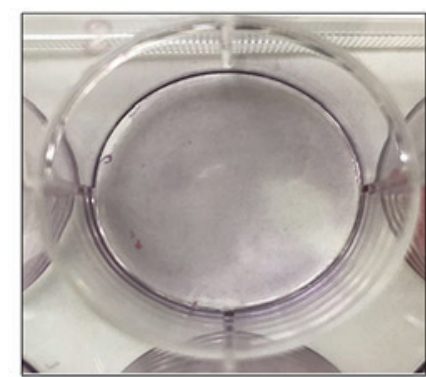

Con

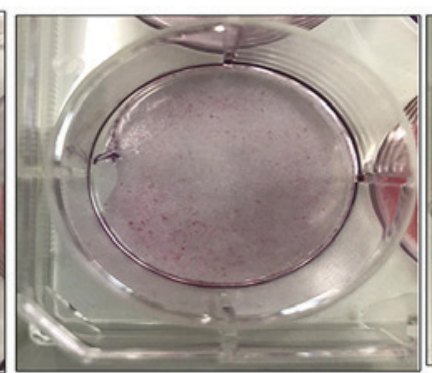

$10^{-5} \mathrm{M}$

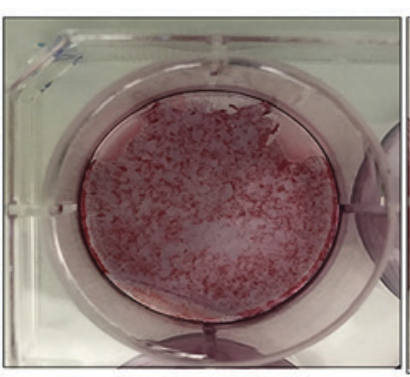

$10^{-6} \mathrm{M}$

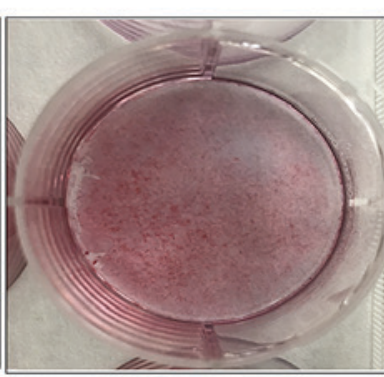

$10^{-7} \mathrm{M}$

Figure 2. VK2 enhances MC3T3-E1 osteoblast differentiation and mineralization. (A) ALP activity was significantly increased in MC3T3-E1 osteoblasts treated with $1 \mu \mathrm{M} \mathrm{VK} 2$ compared with the untreated control, and this was not observed with the other concentrations. ALP activity was even more significantly enhanced in the VK2-treated cells on day 5 compared with the untreated control. (B) Osteoblasts treated with $1 \mu \mathrm{M}$ VK2 had a significantly higher number of alizarin red-stained mineralized nodules compared with untreated control cells. (C) Representative images of alizarin red staining. The experimental data are expressed using the mean \pm standard deviation. Significance analysis of the experimental data for each group was performed using one-way analysis of variance and Tukey's multiple comparisons test. ${ }^{*} \mathrm{P}<0.05,{ }^{* * *} \mathrm{P}<0.01$ vs. respective control group. VK2, vitamin K2; ALP, alkaline phosphatase; Con, control.

VK2 enhances ALP activity and promotes MC3T3-E1 osteoblast differentiation and mineralization. To determine the optimal concentration of VK2 that promotes MC3T3-E1 osteoblast differentiation and mineralization, an ALP assay and alizarin red staining were performed, respectively, following treatment of the cells with $10^{-7}-10^{-5} \mathrm{M} V \mathrm{~V} 2$ for 1-7 days. Treatment with $1 \mu \mathrm{M}$ VK2 significantly increased ALP activity, while other concentrations were not notably effective. In addition, maximum ALP activity with $1 \mu \mathrm{M}$ VK2 was observed on the 5th day (Fig. 2A). Bone formation is accompanied by osteogenic differentiation and mineralization, i.e., calcium deposition. MC3T3-E1 osteoblasts treated with VK2 $(1 \mu \mathrm{M})$ for 7 days exhibited significant calcium deposition, as detected by alizarin red staining (Fig. 2B and C).

VK2 stimulates autophagy in MC3T3-E1 osteoblasts. To analyze the effects of VK2 on autophagy in MC3T3-E1 osteoblasts, the cells were treated with VK2 $(1 \mu \mathrm{M})$ for $0.5-1.5 \mathrm{~h}$ (each day for 7 days), various autophagy markers, including LC3 and Beclin-1, were detected on days 1, 3, 5 and 7 of differentiation. The ratio of LC3II/LC3I is frequently analyzed to determine the extent of autophagy. Treatment with VK2 for 0.5-1.5 h steadily increased LC3I conversion rates in the osteoblasts on days 1, 3, 5 and 7 (Fig. 3A). In addition, VK 2 treatment for $1 \mathrm{~h}$ significantly increased the conversion of LC3I at 1, 3, and 5 days (Fig. 3B-D).
Furthermore, the LC3II/LC3I ratio and Beclin-1 levels were higher on day 5, corresponding to the strongest ALP activity (Fig. 3G). Autophagy was also evaluated using immunofluorescence and MDC staining assays. The number of fluorescent bodies and the intensity increased with VK2 and decreased following treatment with 3-MA+VK2. MDC is a marker for autolysosomes; the results demonstrated that the VK2 group had stronger fluorescence, and the fluorescence intensity of the 3-MA+VK2 group was markedly reduced (Fig. 3H). The inhibitory effect of 3-MA on VK2-induced autophagy was confirmed with the significantly decreased LC3II/LC3I ratio in cells treated with 3-MA, and significantly higher ratio in cells treated with rapamycin. Notably, the conversion rate of LC3II/LC3I in the VK2+3-MA group was lower compared with the VK2 group (Fig. 4A). These results demonstrated that VK2 stimulated autophagy in MC3T3-E1 osteoblasts.

3-MA inhibits the osteogenic differentiation and mineralization induced by $V K 2$. To confirm the role of autophagy in VK2-induced osteogenic differentiation and mineralization, the ALP assay and alizarin red staining were performed on cells treated additionally with 3-MA. The 3-MA treated osteoblasts (3-MA+VK2 group) exhibited significantly reduced ALP activity compared with the VK2 group (Fig. 4B). In addition, 3-MA slightly inhibited the mRNA expression of osteogenic differentiation markers compared with the control 
A

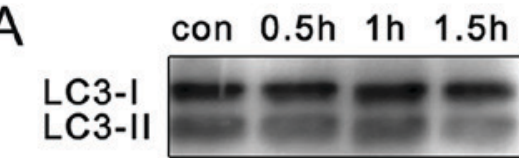

1 day

LC3-I

LC3-II

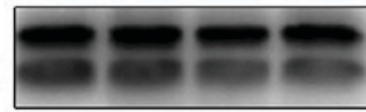

LC3-I

LC3-II

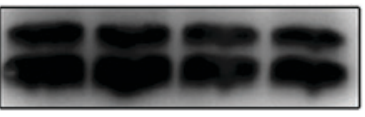

5 days

LC3-I

LC3-II

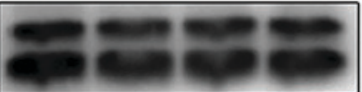

7 days

\section{$\beta$-actin}

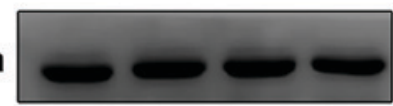

C

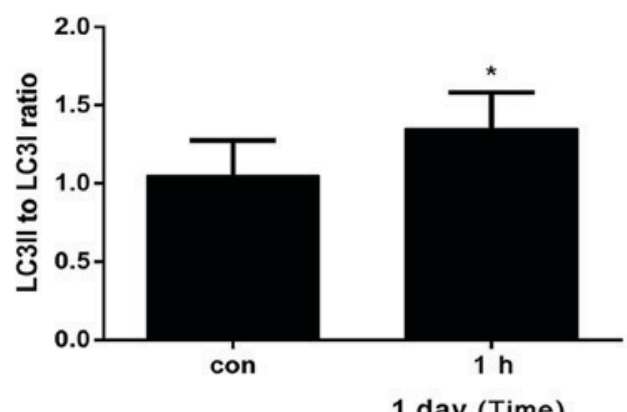

E

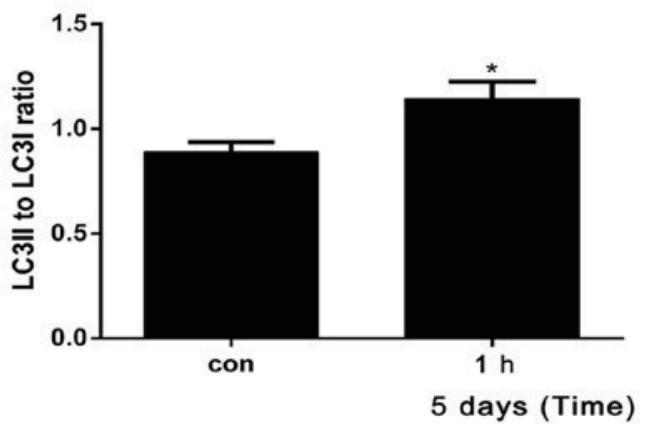

B

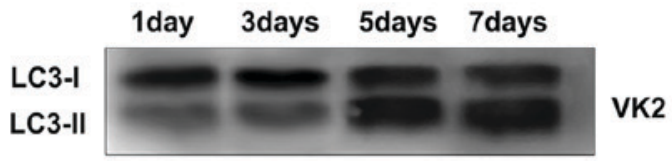

LC3-I LC3-II

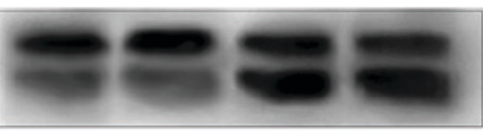
Con

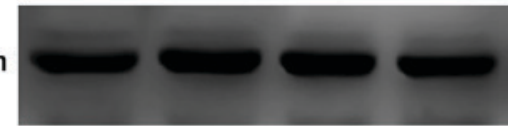

D

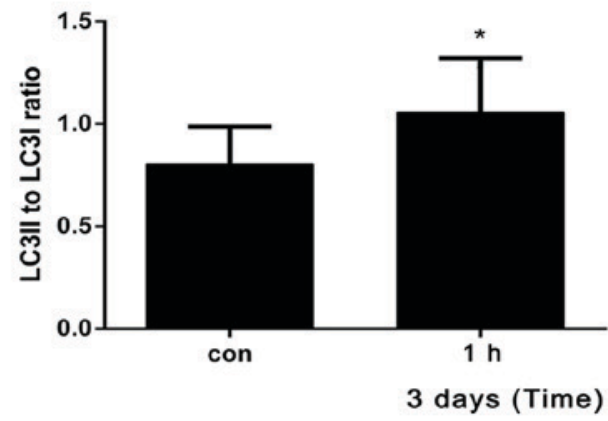

F

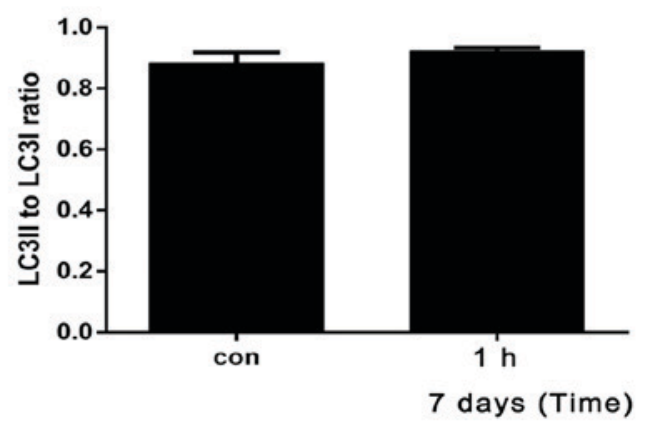

G
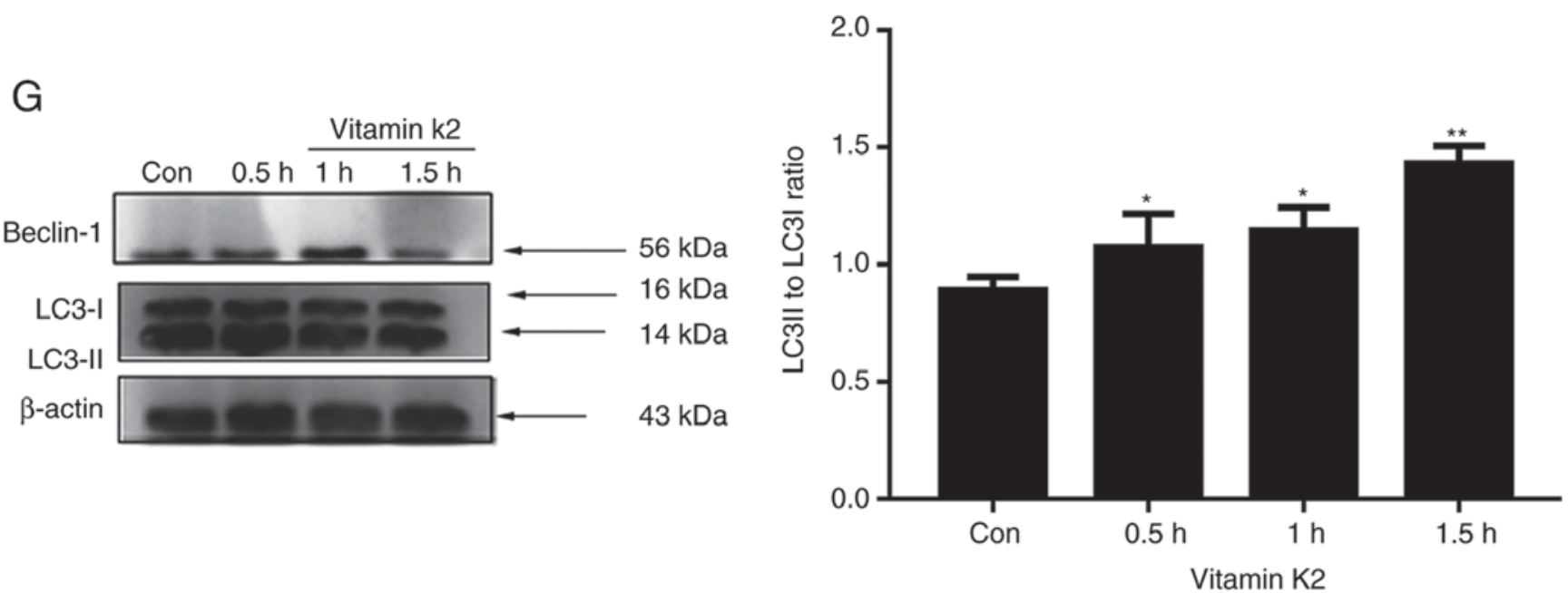

Figure 3. VK2 stimulates autophagy in MC3T3-E1 osteoblasts. The degree of autophagy was evaluated on the basis of LC3I to LC3II conversion (LC3II/LC3I). (A) Treatment with $1 \mu \mathrm{M}$ VK2 for 0.5-1.5 h steadily increased LC3I conversion rates in MC3T3-E1 osteoblasts over (B) 1, 3, 5 and 7 days. Compared with the untreated control cells, those treated with VK2 for $1 \mathrm{~h}$ exhibited a significantly higher degree of autophagy at (C) 1 , (D) 3 and (E) 5 days, although not at (F) 7 days. (G) Higher LC3II/LC3I conversion rates were observed on day 5 after $0.5,1$ and 1.5 h of treatment compared with the untreated controls. 

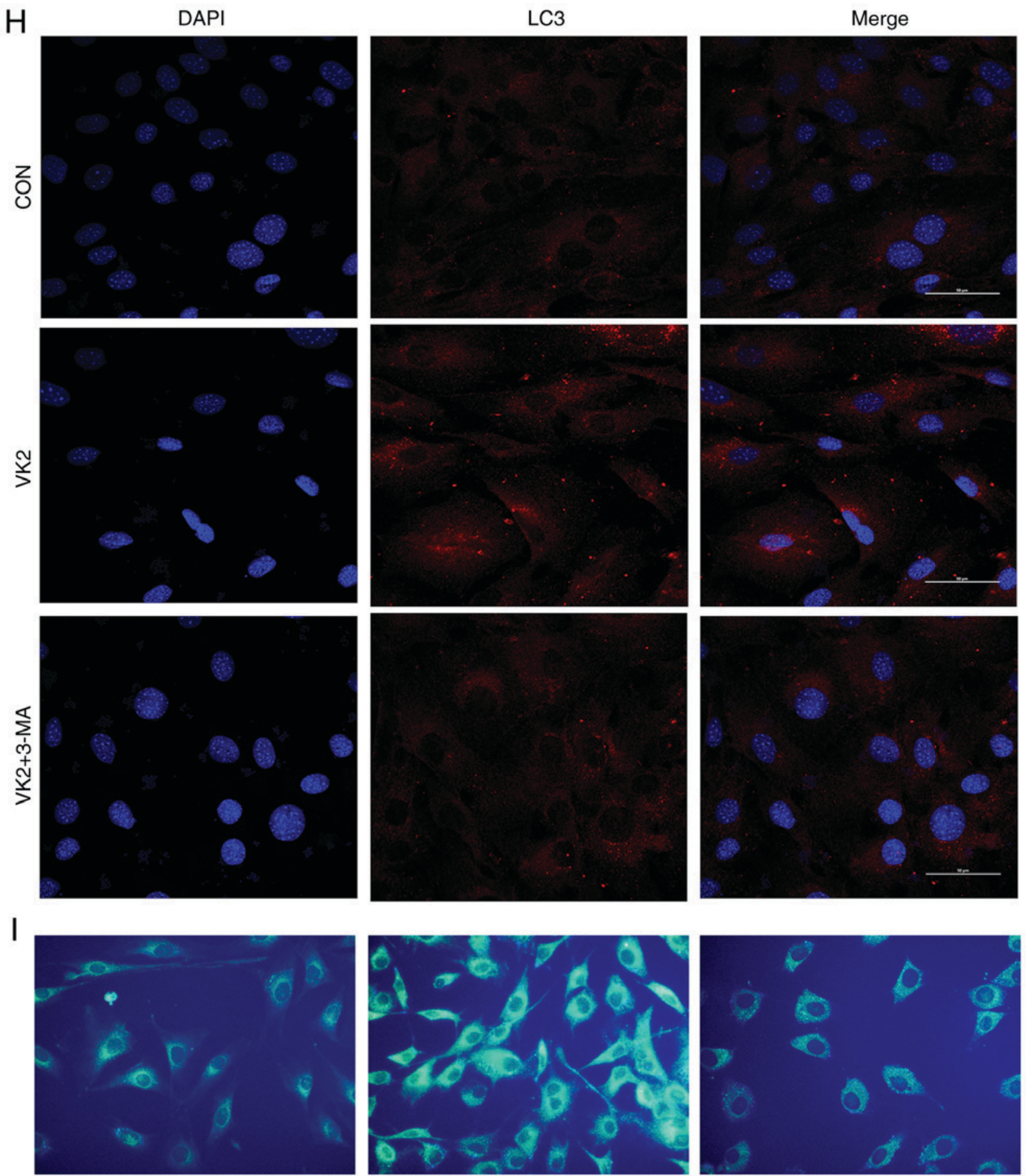

CON

VK2

VK2+3-MA

Figure 3. Continued. (H) To further verify the effect of vitamin K2 on autophagy in MC3T3-E1 osteoblasts, cells were stained in situ with fluorescence labeled anti-LC3b. The number of fluorescent bodies and the fluorescence intensity increased with VK2 and decreased following treatment with 3-MA+VK2 compared with the untreated controls. (I) MDC is a marker for autolysosomes. MDC staining was used to confirm the abundance of autophagic vacuoles in VK2-treated cells. In the control group, weak and diffuse MDC staining was observed throughout the cytoplasm, and very little punctate staining. In VK2-treated cells, the MDC staining was visibly enhanced, and its distribution pattern was altered from diffuse to punctate accumulation. The punctate staining in the 3-MA+VK2 treatment group was markedly reduced. Scale bars, $50 \mu \mathrm{m}$. The experimental data are expressed as the mean \pm standard deviation. Significance analysis of the experimental data for each group was performed using one-way analysis of variance and Tukey's multiple comparisons test. ${ }^{*} \mathrm{P}<0.05$, ${ }^{* * *} \mathrm{P}<0.01$ vs. respective control group. VK2, vitamin K2; ALP, alkaline phosphatase; 3-MA, 3-methyladenine; LC3, microtubule associated protein 1 light chain 3- $\alpha$; Con, control; MDC, monodansylcadaverine.

(Fig. 4C). 3-MA inhibited the mineralization induced by VK2 (Fig. 4D). These results demonstrated that autophagy induced by VK2 stimulation is involved in osteogenic differentiation and mineralization.

\section{Discussion}

MC3T3-E1 osteoblasts were used to study the mechanism of VK2-mediated amelioration of osteoporosis 

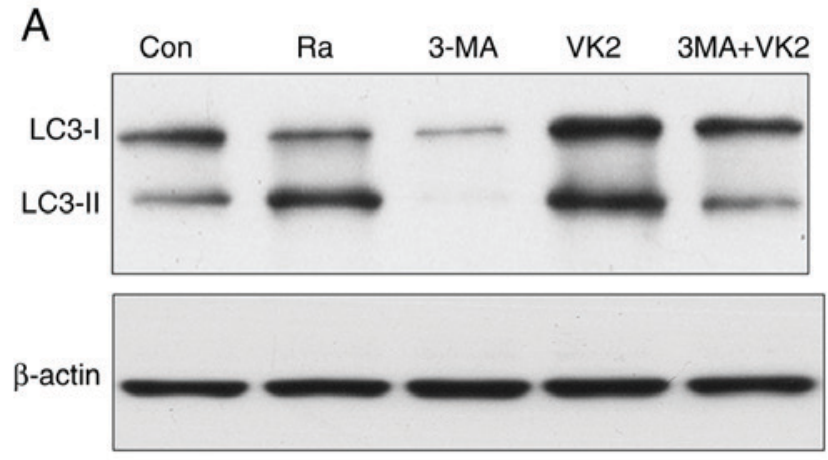

B
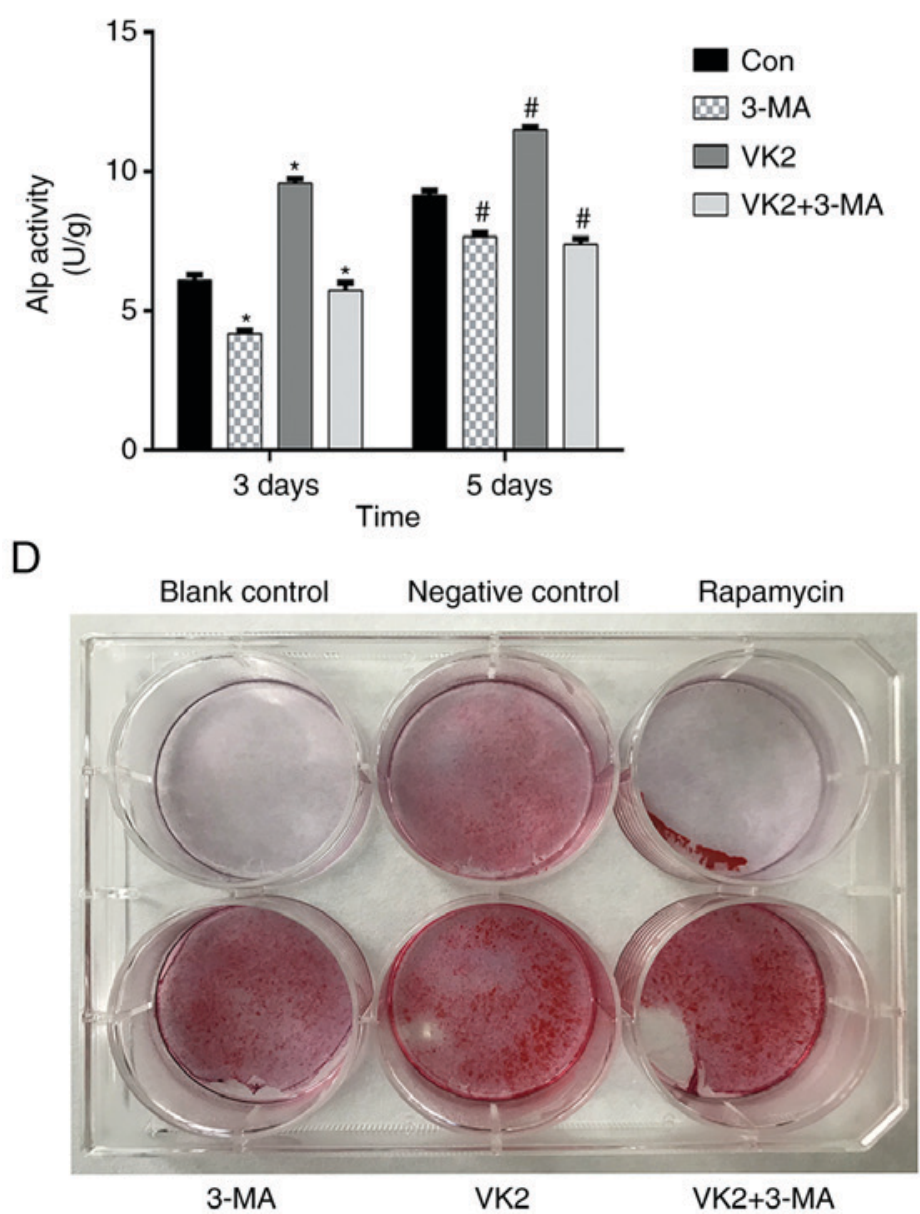

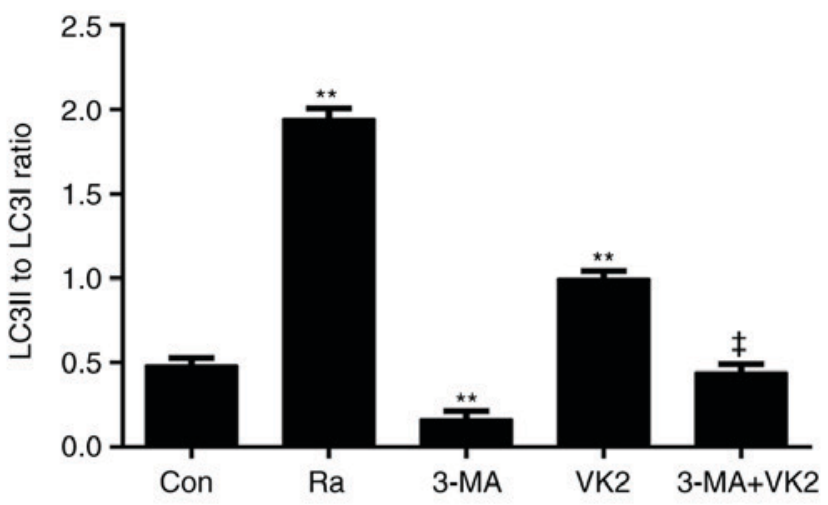

C
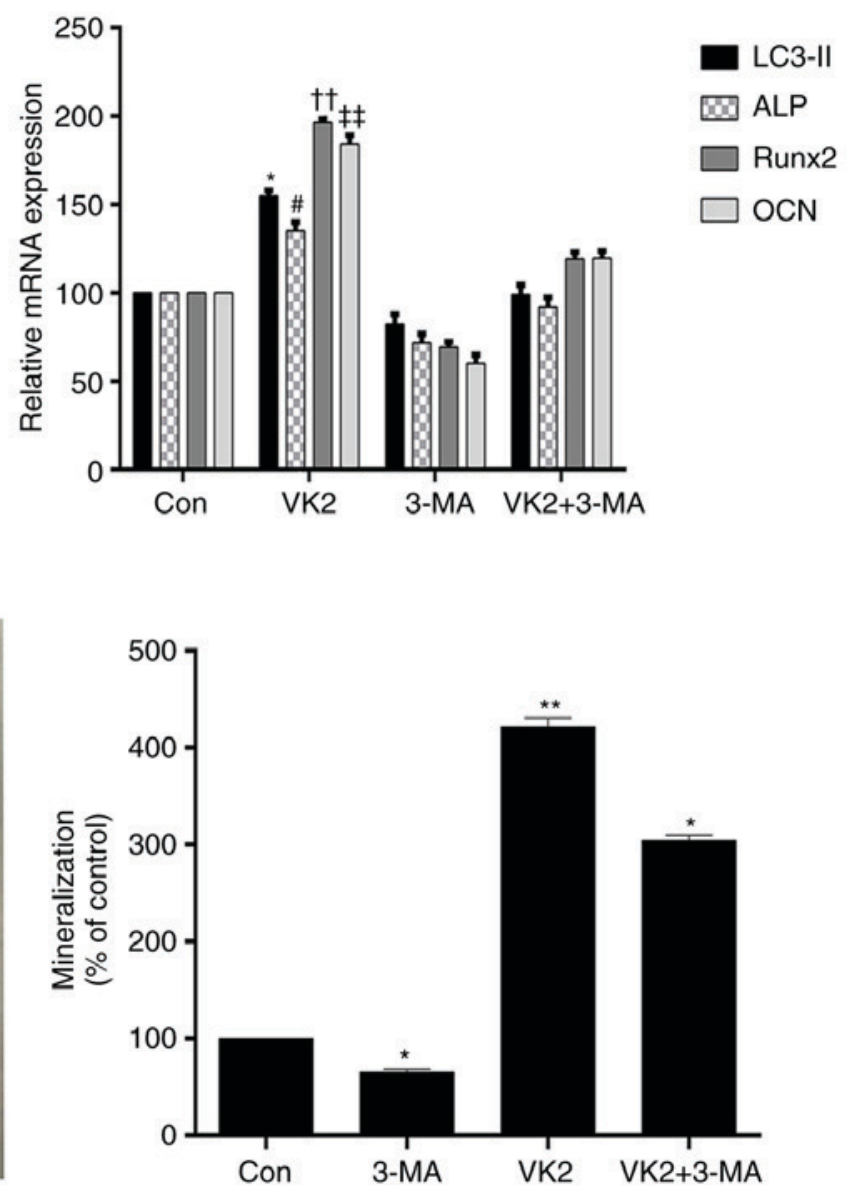

Figure 4. Association between autophagy and differentiation in MC3T3-E1 osteoblasts. (A) To further verify the inhibitory effect of 3-MA on autophagy mediated by VK2, the western blotting results demonstrated that the conversion rate of LC3II/LC3I in cells treated with the autophagy inhibitor 3-MA was significantly decreased, while that of rapamycin was significantly higher. The conversion rate of LC3II/LC3I in VK2+3-MA group was lower compared with the VK2 group. ${ }^{* *} \mathrm{P}<0.01$ vs. control group and ${ }^{\ddagger} \mathrm{P}<0.05$ vs. VK2 group. (B) Compared with the untreated control cells, the 3 -MA treated osteoblasts exhibited a significant inhibition of ALP activity, and the ALP activity of the VK2+3-MA treated cells was significantly lower compared with the VK2-treated cells. ${ }^{*} \mathrm{P}<0.05$ vs. control group at 3 days; ${ }^{*} \mathrm{P}<0.05$ vs. control group at 5 days. (C) The mRNA expression levels of LC3II, ALP, OCN and Runx 2 in VK 2 -treated cells increased. ${ }^{*} \mathrm{P}<0.05$ vs. LC3II control; ${ }^{*} \mathrm{P}<0.05$ vs. ALP control; ${ }^{\dagger} \mathrm{P}<0.01$ vs. Runx 2 control; ${ }^{*} \mathrm{P}<0.01$ vs. OCN control. (D) In addition, alizarin red staining indicated that 3-MA markedly inhibited the osteogenic differentiation induced by VK2. The experimental data are expressed as the mean \pm standard deviation. Significance analysis of the experimental data for each group was performed using one-way analysis of variance and Tukey's multiple comparisons test. ${ }^{*} \mathrm{P}<0.05,{ }^{* *} \mathrm{P}<0.01$ vs. control group. VK2, vitamin K2; 3-MA, 3-methyladenine; Ra, rapamycin; Con, control; ALP, alkaline phosphatase; Runx2, runt related transcription factor 2; OCN, osteocalcin; LC3, microtubule associated protein 1 light chain 3- $\alpha$.

symptoms, as this cell line is routinely used to study the osteogenic characteristics of bone differentiation and mineralization in vitro. Biomarkers including ALP, RUNX2 and OCN are upregulated in the bone matrix during osteogenic differentiation and mineralization, and are thus used to assess the these processes in in vitro models $(20,21)$. Osteoblasts were stimulated with different concentrations of VK2, and osteogenic differentiation and mineralization were evaluated by ALP activity and alizarin red staining, respectively; the concentration of $1 \mu \mathrm{M}$ induced the most 
significant effect on osteoblast differentiation. The present results are consistent with previous studies that have correlated VK2 with increased osteoblast differentiation and mineralization (22-24). However, the specific mechanism of VK2 in promoting bone metabolism has not yet been elucidated. It was demonstrated that VK2 induced autophagy during osteoblast differentiation and mineralization using the markers LC3 and beclin 1. Studies have increasingly demonstrated a role for autophagy in the occurrence and development of osteoporosis. Studies on human genome-wide association data report that autophagy-related genes are associated with osteoporosis $(15,25)$. In addition, the degree of autophagy decreases along with the expression of LC3-II, beclin 1 and unc-51 like autophagy activating kinase 1, while apoptosis and p62 expression increases during osteoporosis $(16,26,27)$. Decreased autophagy likely promotes osteoporosis by increasing oxidative stress $(28,29)$. Furthermore, certain studies have demonstrated that autophagy is associated with the differentiation of osteoblasts. It has been demonstrated that autophagy-associated proteins are involved in osteoblast differentiation and bone formation, and other studies have reported that autophagy affects osteoblast mineralization and bone cell network structure $(30,31)$. Finally, mouse knockout models of autophagy genes illustrate a significant decrease in trabecular bone volume, trabecular number and trabecular thickness $(30,32)$. The present study also confirmed that VK2 may stimulate autophagy in MC3T3 cells to promote differentiation and mineralization.

The use of MC3T3 cells is the primary limitation of the present study; these cells are appropriate for the purpose of this research and numerous studies have used MC3T3-E1 to assess differentiation and mineralization (33-36). However, there are limitations associated with performing experiments with a single cell line. The present results may be confirmed by using an additional cell line, such as mesenchymal stem cells, and additional factors may be used to evaluate autophagy in the future, including other types of autophagy inhibitors to exclude off-target effects, and in vivo experiments. Finally, the specific pathways associated with autophagy were completely explored. Therefore, more in-depth mechanistic studies are required.

In conclusion, the present findings indicated that VK2 promotes autophagy during the differentiation and mineralization of osteoblasts, which may be a potential therapeutic target for osteoporosis.

\section{Acknowledgements}

Not applicable.

\section{Funding}

This study was supported by the Science and Technology Project of Shenyang (grant no. F16-102-4-00).

\section{Availability of data and materials}

All data generated or analyzed during this study are included in this published article.

\section{Authors' contributions}

WL and QL conceived and designed the study. WL, SZ and YL performed the experiments. WL and JL analyzed the data. WL was a major contributor in writing the manuscript. All authors read and approved the final manuscript.

\section{Ethics approval and consent to participate}

Not applicable.

\section{Patient consent for publication}

Not applicable.

\section{Competing interests}

The authors declare that they have no competing interests.

\section{References}

1. Tella SH and Gallagher JC: Prevention and treatment of postmenopausal osteoporosis. J Steroid Biochem Mol Biol 142: 155-170, 2014.

2. Glaser DL and Kaplan FS: Osteoporosis. Definition and clinical presentation. Spine (Phila Pa 1976) 22 (24 Suppl): 12S-16S 1997.

3. Edwards MH, Dennison EM, Aihie Sayer A, Fielding R and Cooper C: Osteoporosis and sarcopenia in older age. Bone 80: 126-130, 2015.

4. Diab DL and Watts NB: Postmenopausal osteoporosis. Curr Opin Endocrinol Diabetes Obes 20: 501-509, 2013.

5. Clarke BL: Anti-sclerostin antibodies: Utility in treatment of osteoporosis. Maturitas 78: 199-204, 2014.

6. Levine JP: Identification, diagnosis, and prevention of osteoporosis. Am J Manag Care 17 (Suppl 6): S170-S176, 2011.

7. Palermo A, Tuccinardi D, D'Onofrio L, Watanabe M, Maggi D, Maurizi AR, Greto V, Buzzetti R, Napoli N, Pozzilli P and Manfrini S: Vitamin K and osteoporosis: Myth or reality? Metabolism 70: 57-71, 2017

8. Nelsestuen GL,Zytkovicz TH and Howard JB: The mode of action of vitamin K. Identification of gamma-carboxyglutamic acid as a component of prothrombin. J Biol Chem 249: 6347-6350, 1974.

9. Stenflo J, Fernlund P, Egan W and Roepstorff P: Vitamin K dependent modifications of glutamic acid residues in prothrombin. Proc Natl Acad Sci USA 71: 2730-2733, 1974.

10. Berkner KL: The vitamin K-dependent carboxylase. Annu Rev Nutr 25: 127-149, 2005

11. Hauschka PV, Lian JB and Gallop PM: Direct identification of the calcium-binding amino acid, gamma-carboxyglutamate, in mineralized tissue. Proc Natl Acad Sci USA 72: 3925-3929, 1975.

12. Coutu DL, Wu JH, Monette A, Rivard GE, Blostein MD and Galipeau J: Periostin, a member of a novel family of vitamin $\mathrm{K}$-dependent proteins, is expressed by mesenchymal stromal cells. J Biol Chem 283: 17991-18001, 2008.

13. Rubinacci A: Expanding the functional spectrum of vitamin $\mathrm{K}$ in bone. Focus on: 'Vitamin K promotes mineralization, osteoblast to osteocyte transition, and an anti-catabolic phenotype by \{gamma\}-carboxylation-dependent and -independent mechanisms'. Am J Physiol Cell Physiol 297: C1336-1338, 2009.

14. Atkins GJ, Welldon KJ, Wijenayaka AR, Bonewald LF and Findlay DM: Vitamin K promotes mineralization, osteoblast-to-osteocyte transition, and an anticatabolic phenotype by \{gamma\}-carboxylation-dependent and -independent mechanisms. Am J Physiol Cell Physiol 297: C1358-1367, 2009.

15. Pierrefite-Carle V, Santucci-Darmanin S, Breuil V, Camuzard O and Carle GF: Autophagy in bone: Self-eating to stay in balance. Ageing Res Rev 24: 206-217, 2015.

16. Parzych KR and Klionsky DJ: An overview of autophagy: Morphology, mechanism, and regulation. Antioxid Redox Signal 20: 460-473, 2014.

17. White E, Mehnert JM and Chan CS: Autophagy, Metabolism, and Cancer. Clin Cancer Res 21: 5037-5046, 2015. 
18. Mizushima N and Levine B: Autophagy in mammalian development and differentiation. Nat Cell Biol 12: 823-830, 2010.

19. Mizushima N, Levine B, Cuervo AM and Klionsky DJ: Autophagy fights disease through cellular self-digestion. Nature 451: 1069-1075, 2008.

20. Yamaguchi M: The role of regucalcin in bone homeostasis: Involvement as a novel cytokine. Integr Biol (Camb) 6: 258-266, 2014.

21. Raouf A and Seth A: Ets transcription factors and targets in osteogenesis. Oncogene 19: 6455-6463, 2000.

22. Yamaguchi M and Weitzmann MN: Vitamin K2 stimulates osteoblastogenesis and suppresses osteoclastogenesis by suppressing NF- $\kappa$ B activation. Int J Mol Med 27: 3-14, 2011.

23. Ichikawa T, Horie-Inoue K, Ikeda K, Blumberg B and Inoue S: Vitamin K2 induces phosphorylation of protein kinase A and expression of novel target genes in osteoblastic cells. J Mol Endocrinol 39: 239-247, 2007.

24. Zhang YL, Yin JH, Ding H, Zhang W, Zhang CQ and Gao YS Protective effect of VK2 on glucocorticoid-treated MC3T3-E1 cells. Int J Mol Med 39: 160-166, 2017.

25. Nollet M, Santucci-Darmanin S, Breuil V, Al-Sahlanee R, Cros C, Topi M, Momier D, Samson M, Pagnotta S, Cailleteau L, et al: Autophagy in osteoblasts is involved in mineralization and bone homeostasis. Autophagy 10: 1965-1977, 2014.

26. Chen K, Yang YH, Jiang SD and Jiang LS: Decreased activity of osteocyte autophagy with aging may contribute to the bone loss in senile population. Histochem Cell Biol 142: 285-295, 2014.

27. Pan F, Liu XG, Guo YF, Chen Y, Dong SS, Qiu C, Zhang ZX, Zhou Q, Yang TL, Guo Y, et al: The regulation-of-autophagy pathway may influence Chinese stature variation: Evidence from elder adults. J Hum Genet 55: 441-447, 2010.

28. Jilka RL, Weinstein RS, Parfitt AM and Manolagas SC: Quantifying osteoblast and osteocyte apoptosis: Challenges and rewards. J Bone Miner Res 22: 1492-1501, 2007.

29. Li L, Tan J, Miao Y, Lei P and Zhang Q: ROS and Autophagy: Interactions and molecular regulatory mechanisms. Cell Mol Neurobiol 35: 615-621, 2015.
30. Liu F, Fang F, Yuan H, Yang D, Chen Y, Williams L, Goldstein SA, Krebsbach PH and Guan JL: Suppression of autophagy by FIP200 deletion leads to osteopenia in mice through the inhibition of osteoblast terminal differentiation. J Bone Miner Res 28: 2414-2430, 2013.

31. Xi G, Rosen CJ and Clemmons DR: IGF-I and IGFBP-2 stimulate AMPK activation and autophagy, which are required for osteoblast differentiation. Endocrinology 157: 268-281, 2016.

32. Chang KH, Sengupta A, Nayak RC, Duran A, Lee SJ, Pratt RG, Wellendorf AM, Hill SE, Watkins M, Gonzalez-Nieto D, et al: p62 is required for stem cell/progenitor retention through inhibition of IKK/NF-kB/Ccl4 signaling at the bone marrow macrophage-osteoblast niche. Cell Rep 9: 2084-2097, 2014

33. Liu Y, Huang L, Hao B, Li H, Zhu S, Wang Q, Li R, Xu Y and Zhang X: Use of an osteoblast overload damage model to probe the effect of icariin on the proliferation, differentiation and mineralization of MC3T3-E1 cells through the Wnt/ $\beta$-catenin signalling pathway. Cell Physiol Biochem 41: 1605-1615, 2017.

34. Kim EC, Kim TH, Jung JH, Hong SO and Lee DW: Enhanced osteogenic differentiation of MC3T3-E1 on rhBMP-2-immobilized titanium via click reaction. Carbohydr Polym 103: 170-178, 2014.

35. He Y, Wang S, Mu J, Dai L, Zhang Z, Sun Y, Shi W and Ge D: Synthesis of polypyrrole nanowires with positive effect on MC3T3-E1 cell functions through electrical stimulation. Mater Sci Eng C Mater Biol Appl 71: 43-50, 2017.

36. Mu W, Wang Z, Ma C, Jiang Y, Zhang N, Hu K, Li L and Wang Z: Metformin promotes the proliferation and differentiation of murine preosteoblast by regulating the expression of sirt6 and oct4. Pharmacol Res 129: 462-474, 2018.

This work is licensed under a Creative Commons Attribution-NonCommercial-NoDerivatives 4.0 International (CC BY-NC-ND 4.0) License. 